\title{
Clinical research networks: a step towards evidence-based practice in pediatric rheumatology
}

\author{
Elizabeth D Mellins and Lisa G Rider
}

Pediatric rheumatologists are often forced to make clinical decisions in the absence of robust evidence about the most effective therapies for their patients. A number of factors have contributed to the relative lack of practice-guiding information. Of an estimated 200,000 children with rheumatic diseases in the US, $30 \%$ are under the care of pediatric rheumatologists, and it is likely that an equal number see adult rheumatologists (Mayer ML et al. [2003] Arthritis Rheum 49: 759-765). With this dispersion of a relatively small patient population, adequately-powered studies can rarely be accomplished at a single site. The number of investigators appropriately positioned to carry out clinical research in pediatric rheumatology is also small. In total, there are 215 board-certified pediatric rheumatologists in the US; 13 states don't have a pediatric rheumatologist at all. Many pediatric rheumatologists are 'one-person divisions' with substantial clinical responsibility. In addition to these barriers to investigator-initiated studies, pharmaceutical companies have historically seen the pediatric rheumatology population as unattractively small or too high risk. FDA policies to stimulate studies in children apply to pediatric forms of nonorphan diseases; only polyarticular juvenile idiopathic arthritis (JIA) qualifies and has benefited from increased industry-sponsored trials.

Scarcity of evidence also confronts clinicians in many other pediatric subspecialties. The striking counter-example is pediatric oncology. Key to this difference was the initiation in 1955 of multi-institutional clinical investigation, sponsored by the National Cancer Institute $(\mathrm{NCl})$ of the $\mathrm{NIH}$. Four groups that focused on pediatric cancers have merged to form the Children's Oncology Group (COG), with member institutions in North America, Europe, and Australia. COG physicians, using protocol-based clinical care, systematically determine optimal available treatments. Studies show a significant improvement in outcome for children treated using network protocols in participating centers;
The recent

increase in

multicenter

research in

pediatric

rheumatology

demonstrates

the commitment

of the community

to a [systematic]

approach

ED Mellins is a member of the Nature Clinical

Practice Rheumatology

Advisory Board. She

is Associate Professor

in the Department of

Pediatrics at Stanford

University School of

Medicine, Stanford, CA,

USA and was a founder

of CARRA. LG Rider

is the Deputy Chief

of the Environmental

Autoimmunity Group of

the National Institute of

Environmental Health

Sciences, NIH, Bethesda,

$M D$, USA, and was a

founder of IMACS.

\section{Competing interests}

ED Mellins declared she

has no competing interests.

LG Rider has declared

associations with the

following organizations:

the American College of

Rheumatology, the Cure JM

foundation, The Myositis

Association and the National

Institute of Environmental

Health Sciences. See the

article online for full details of

these relationships.

www.nature.com/clinicalpractice doi:10.1038/ncprheum0405 consequently, $90 \%$ of US children with cancer now are treated according to a COG protocol (Liu Let al. [2003] Cancer 97: 1339-1345).

The recent increase in multicenter research in pediatric rheumatology demonstrates the commitment of the community to a COGlike approach. The Pediatric Rheumatology Collaborative Study Group (PRCSG) and the Childhood Arthritis and Rheumatology Research Alliance (CARRA) in North America, the EU-supported Paediatric Rheumatology International Trials Organisation (PRINTO) in Europe, and smaller disease-specific groups like the International Myositis Assessment and Clinical Studies Group (IMACS) encourage scientific exchange and collaborative research. These initiatives are producing new consensus tools for measuring disease activity and remission for pediatric arthritis, myositis, and vasculitis, and have facilitated pivotal drug trials in JIA, pediatric lupus, and juvenile dermatomyositis.

What factors will contribute to the optimum use of these networks? Families need educating about the importance of participation in clinical studies and therapeutic trials. Conducting research in the context of clinical care requires appropriate infrastructure (e.g. personnel, state-of-the-art data-collection systems) and some changes in the culture of clinical practice. Active outreach to all physicians taking care of children with rheumatic disease will increase the patient research base and ensure more efficient transfer of new information into widespread clinical practice. A critical mass of clinical researchers must be nurtured from both within and outside the pipeline of trainees in pediatric rheumatology (there are currently 65 training fellows in the US). The substantial funds needed to meet these objectives will probably require that government, private foundations, and industry all contribute. Realizing the potential of the clinical networks presents significant challenges, but holds the promise of great benefit for our young patients. 